\title{
Impact of the COVID-19 pandemic on Otolaryngology trainee education
}

\author{
Ehab Y. Hanna, MD, FACS ${ }^{1}$ \\ ${ }^{1}$ The University of Texas MD Anderson Cancer Center
}

June 22, 2020

\begin{abstract}
Background The COVID-19 pandemic has reduced clinical volume with a negative impact on trainee education. Methods Survey study of Otolaryngology trainees in North America, during the COVID-19 pandemic in April $2020 . \quad$ Results Of 216 respondents who accessed the survey, 175 (83\%) completed the survey. Respondents reported a universal decrease in clinical activities (98.3\%). Among participants who felt their program utilized technology well, there were significantly decreased concerns to receiving adequate educational knowledge $(29.6 \%$ vs. $65.2 \%, \mathrm{p}=0.003)$. However, $68 \%$ of trainees still expressed concern in ability to receive adequate surgical training. In addition, $54.7 \%$ of senior trainees felt that the pandemic had a negative impact on their ability to secure a job or fellowship after training. Conclusions Trainees universally felt a negative impact due to the COVID-19 pandemic. Use of technology was able to alleviate some concerns in gaining adequate educational knowledge, but decreased surgical training remained the most prevalent concern.
\end{abstract}

\section{Theresa Guo, MD, ${ }^{1}$ Kimberley L Kiong, MBBS, ${ }^{1}$ Christopher MKL Yao, MD, ${ }^{1}$ Melina Windon, MD, ${ }^{2}$ Denna Zebda, MD, ${ }^{3}$ Yelda Jozaghi, MD, ${ }^{1}$ Xiao Zhao, MD, PhD, ${ }^{1}$ Amy Hessel, MD, ${ }^{1}$ Ehab Hanna, MD, FACS ${ }^{1}$}

\footnotetext{
${ }^{1}$ Department of Head \& Neck Surgery, The University of Texas MD Anderson Cancer Center, Houston, TX, United States

${ }^{2}$ Department of Otolaryngology-Head \& Neck Surgery, Johns Hopkins University School of Medicine, Baltimore, MD, United States

${ }^{3}$ Department of Otorhinolaryngology-Head \& Neck Surgery, McGovern Medical School, The University of Texas Health Science Center at Houston, Houston, TX, United States
}

Funding: 2T32DC000027-26 (MW)

Corresponding Author: Ehab Hanna, MD University of Texas MD Anderson Cancer Center 1400 Pressler Street Houston, TX 77030 Office: (713)-835-1001 Fax: (713)-794-4662 E-mail: eyhanna@mdanderson.org

Running title: Impact of COVID-19 on Otolaryngology education

Key words: Resident education; surgical training; COVID-19; didactic education; career planning

\section{Introduction}


Due to the COVID-19 pandemic, there has been a significant decrease in clinical activities, reduction of elective surgeries, and restriction of workforce to essential personnel in hospitals nationwide including among Otolaryngology departments. ${ }^{1}$ Otolaryngology programs have faced significant reductions in surgical case volume to conserve resources, with many institutions limiting trainee coverage. ${ }^{2-4}$ In addition, some trainees have been redeployed to practice outside of their specialty to meet the rising need for COVID-19 related medical care. ${ }^{5}$ As these parameters only begin to ease, their impact on education and specifically surgical training is limited, ${ }^{6,7}$ and questions have been raised as to whether surgical training will need to be extended to ensure adequacy. 6,8

In Otolaryngology, concerns regarding reduction in surgical and specialty-specific clinical training are layered with concerns regarding personal safety and protection. Among healthcare providers, otolaryngologists have been reported as among the highest risk for exposure and infection due to close contact with mucous membranes that may contain high viral load ${ }^{9,10}$ and performance of high risk procedures such as mucosal surgery and tracheostomy. ${ }^{11}$

In the setting of social distancing, didactic teaching sessions have moved to virtual classroom and lecture models. ${ }^{8,12}$ Models of online education, such as the Global On-Line Fellowship program, have shown the potential efficacy of remote learning within Otolaryngology-Head and Neck Surgery. ${ }^{13}$ In addition to changes in clinical experiences, national gatherings that contribute to trainee education and career advancement including national conferences, oral board examinations, and accreditation testing have been either postponed or cancelled. ${ }^{6,8}$ Lastly, some trainees are preparing to begin their careers at a time of uncertainty both for the healthcare system and the economy.

A recent resident survey during the COVID-19 area showed that $73 \%$ residents continue to feel concerned about contracting the virus and its impact on their training. ${ }^{4}$ To further explore the impact of the COVID-19 pandemic on Otolaryngology trainee education and impact on future career, we performed a survey of Otolaryngology residents and fellows in the United States (US) and Canada regarding educational experiences, use of technology and related concerns surrounding safety and future career during the COVID-19 pandemic.

\section{Materials \& Methods}

After exploratory discussions with trainees at differing levels of training, a 45-question survey was generated to evaluate changes in Otolaryngology trainee experiences related to participation in clinical activities, concerns regarding safety and future career. IRB exemption was obtained through MD Anderson Institutional Review Board through expedited review. Responses were binary (yes or no) or measured on a 5-point Likert scale, with select open ended questions regarding impact on future career, greatest challenges, and skills gained. Additional demographic information including trainee level, length of training program and geographic location was collected. Geographic location included broad regions within the United States, based on US census regions and Canada. Participation was voluntary and solicited electronically. Program directors from the US and Canada were invited to share the survey to their trainees. To maintain anonymity and compile adequate data for evaluation, a minimum of 100 survey results were sought. Survey responses were collected between April 14-21, 2020, during the COVID-19 pandemic.

Descriptive statistics were performed on aggregated survey results. Subgroups were analyzed based on geographic location. Geographic location was considered to be a high COVID-19 region if there were on average greater than 100 COVID-19 cases per 100,000 persons, captured April 21, 2020 (Supplemental Table 1). In addition, subgroups were analyzed based on trainee level (Junior, PGY 1-3, compared to senior level trainees, PGY 4-5 \& fellows). Likert scale responses were treated as ordinal categorical variables. Comparisons were made among answers and respondent characteristics using Pearson's Chi-squared test. Correction for multiple testing was performed using the Benjamini-Hochberg method. Statistical analysis was performed using Microsoft Excel (2016, Microsoft Corporation, Seattle, WA) and Stata version 14.1 (StataCorp, College Station, TX).

\section{Results}


A total of 216 trainees accessed the survey, and 175 respondents (83\%) completed the online survey, including 159 residents and 16 fellows (Table 1), and all regions of the continental US, and Canada were represented (Figure 1). Half of the respondents $(88,50.3 \%)$ responded from high COVID-19 geographic locations.

\section{Clinical activities}

Nearly all participants (98.3\%) reported an overall decrease in clinical activity at their training hospital. Likewise, $97 \%$ reported a reduction in their involvement with in-person clinic visits and $94 \%$ reported a reduction in participation in the operating room (OR). Most trainees (64\%) reported that they were not participating in any in-person clinic visits, though $25 \%$ of respondents reported some participation in telemedicine visits. Fewer participants reported a reduction in inpatient and on-call duties (54.3\%) with $33.7 \%$ reporting an increase in these duties.

\section{Non-clinical educational activities}

A majority $(62.3 \%)$ of participants reported an increase in didactic educational activities and $65.7 \%$ reported that their program had instituted a required educational curriculum (Table 2). Interestingly, a significantly higher proportion of participants in high COVID-19 regions reported an increase in didactic activities $(\mathrm{p}=0.011)$ and a required curriculum $(\mathrm{p}=0.035)$. Most participants $(86.9 \%)$ felt their department utilized technology to good effect for educational activities, and again this was higher among participants from high COVID-19 regions (95.4\% vs. $78.2 \%, \mathrm{p}=0.011)$. Educational activities included participation in collaborative multi-institutional educational efforts (61.7\%) as well as institution based education (62.3\%). Tools utilized during social distancing included Zoom \& WebEx for lectures including collaborative consortiums, resident lectures and journal clubs. Additional electronic resources used included OtoSim, AAO courses, COCLIA, Headmirror podcasts and online textbook reading.

With regard to conducting research, about one third of participants (29.1\%) reported a reduction in research activities, a third reported no change, and a third reported an increase in research activity (34.9\%). A greater proportion of participants reported a reduction in research activities if their research prior to the pandemic involved recruitment of clinical subjects (71.4\%) or laboratory-based research $(71.4 \%)$.

Most respondents felt that their education during the pandemic was either extremely or very important $(57 \%)$ or somewhat important $(30.3 \%)$, while $13 \%$ felt that their education was not so important during the pandemic. This did not differ by training level $(\mathrm{p}=0.10)$, but participants in high COVID-19 areas trended towards a stronger feeling that their education was either extremely or very important $(65.9 \%$ high COVIDvs. $47.1 \%$ low COVID, $\mathrm{p}=0.072$ ).

Despite acknowledging the importance of education, the majority (89.7\%) of respondents felt that their education and training had been negatively impacted by the pandemic. In particular, two-thirds of participants were concerned about receiving adequate surgical training. Smaller proportions of participants expressed concern about receiving adequate training in clinical decision making (42.9\%) and educational knowledge $(34.3 \%)$. Concerns regarding adequate training did not significantly differ by trainee level, however, there was a trend towards fewer senior level trainees expressing concerns for adequate training (Table 2). Notably, 29.1\% ( $\mathrm{n}=51$ ) of participants expressed concern in their ability to complete key indicator cases needed to graduate, and these concerns were acknowledged similarly by both junior (27.2\%) and senior residents $(39.2 \%, \mathrm{p}=0.116$, fellows excluded).

Participants in high COVID-19 regions reported less concern about receiving adequate educational knowledge ( $23.8 \%$ vs. $44.8 \%, \mathrm{p}=0.025)$ and a trend towards less concern in receiving adequate training in clinical decision making ( $32.9 \%$ vs. $52.9 \%, \mathrm{p}=0.062)$ compared to participants in low COVID-19 regions. These differences were not seen when examining concerns regarding adequate surgical training.

Concerns regarding adequate educational knowledge were decreased (29.6\% vs. $65.2 \%)$ among participants who felt their program was able to utilize technology well $(\mathrm{p}=0.003)$, though not significantly correlated with whether a program had a required educational curriculum $(\mathrm{p}=0.250)$, or participation in collaborative 
education effort ( $\mathrm{p}=0.403)$. Use of technology was not associated with concerns regarding adequate training in clinical decision-making $(\mathrm{p}=0.199)$ or surgical training $(\mathrm{p}=0.690)$.

\section{Safety and other concerns}

The majority of trainees reported that they were provided adequate PPE for participation both in the operating room $(81 \%)$ and in the inpatient or ER setting (82.3\%, Table 3). However, $43 \%$ still reported that they also acquired their own PPE. Most participants (76\%) felt safe and protected performing their clinical duties, and $85 \%$ of respondents felt that their department was supportive of suggestions and feedback. While trainees overall felt supported by their departments, some major concerns included exposure to the virus $(70 \%)$, infecting others with the virus $(87.3 \%)$, and the safety of co-workers $(72.8 \%)$. Challenges that trainees reported facing included "changing policies and guidelines" surrounding PPE, "lack of transparency of leadership". Additional challenges included "coping with collective and personal anxiety", "feeling not useful", "dealing with the unknown" and hoping for more "mental health resources." The most frequent challenges cited (half of respondents) were related to reduced "surgical training," decreased clinical experience, and junior residents not being able to participate in OR or inpatient encounters.

\section{Impact on future}

Participants were asked to report their level of concern regarding a variety of additional factors. $59 \%$ of trainees expressed feeling worried about "not receiving enough training before I graduate," and 43.9\% expressed concerns about their ability to achieve future career goals. Cancellation of training courses and conferences directly impacted $60-70 \%$ of participants. For those nearing the completion of training, $16.6 \%$ reported that they were impacted by cancellation or rescheduling of interviews, and $23.4 \%$ were impacted by change in qualifying exam schedules. A third of respondents (35.3\%) felt that the pandemic has had a negative impact on their ability to secure a job or fellowship at the completion of training, and $14.9 \%$ reported that the pandemic had impacted decisions regarding their future. These concerns were more prevalent in senior level trainees, with a majority $(54.7 \%)$ reporting a negative impact on their ability to secure a job or fellowship (Table 3). These impacts included loss of opportunities ("decreased job availability"; "missing out on a full rotation"; "diminished surgical experience ... will not be able to perform [specific] cases as a generalist, previously had aimed to"), considering fellowship training versus general ENT practice ("was considering private practice... (will need to) see what economics of the practices will look like"; "considering if fellowship will be necessary to increase ... exposure to surgical cases"), considering private practice vs. academic practice ("less likely to go to an academic institution"; "academics [have] more financial protection") and needing to delay next steps ("have to delay my fellowship"; "impact my career timeline").

\section{Discussion}

Otolaryngology trainees have been faced with the challenge of achieving clinical knowledge and technical competency in the midst of lost clinical volume and new threats to personal safety caused by the COVID19 pandemic. Herein, we report the results of a survey of North American Otolaryngology residents and fellows, providing additional insight into their perspective on the effects of the pandemic on their education, safety, and future. Building on current published reports, ${ }^{4}$ we found a near-unanimous reduction in clinical volume, a dominant feeling that the pandemic has negatively affected surgical training as has been previously reported. ${ }^{4}$ In addition, we found that use of technology in high COVID prevalent areas was able to alleviate some concerns in receiving adequate educational knowledge and further describe how the pandemic may impact future career.

Otolaryngology programs have experienced vast reductions in surgical case load in response to the American College of Surgeons and the Surgeon General's encouragement for cancellation of elective cases. ${ }^{1,4,14}$ Atop a lighter case load, resident participation in the operating room (OR) at more than $60 \%$ of institutions in a recent survey limiting cases to faculty alone or senior level trainees. ${ }^{4}$ Two-thirds of survey participants expressed concern regarding their ability to receive adequate surgical training, representing one of the most prevalent concerns across trainee level and geographic area. This apprehension appeared to be more closely related to personally developing surgical expertise rather than fulfilling administrative case requirements, 
as concern around ability to graduate and complete key indicator cases were only around half as prevalent. Indeed, a survey of surgical head and neck fellows showed $82 \%$ had already reached adequate case numbers for certification by mid-April of $2020 .{ }^{15}$ The ACGME issued a special communication to address resident concerns regarding training requirements in the current context wherein the reduction in elective operations and the potential for training extension was recognized. Graduation of trainees will be based on the discretion of the program director of the trainee's surgically competency. ${ }^{7,16}$

A lower proportion (43\%) of participants were concerned about training in clinical decision-making. Although almost all participants acknowledged a reduction in in-person clinic participation, about a quarter of respondents reported participation in telemedicine visits. For trainees, telemedicine visits allow for engagement in patient care, and familiarity with a new patient care setting that may be a part of their future practice. With the reduction in surgical caseload, the inpatient census has likewise seen a decrease. Half of trainees in the survey reported reduced participation in call or inpatient duties, which remain a critical aspect of training. ${ }^{17,18}$ Call duties present new challenges to personal safety, with potential high risk exposures through performance of aerosol generating procedures, where residents are at the frontline of initial evaluation. ${ }^{3,19}$ If adequate PPE is not immediately available, trainees must weight their duty to patients with a duty to protect themselves from undue risk of harm. ${ }^{20}$ Given the risk of aerosol generation during common otolaryngology procedures including flexible laryngoscopy and mucosal surgery, multiple sources have recommended the use of at least N95 or equivalent mask, surgical masks, gown, gloves and eye protection. ${ }^{21,22}$ Some institutions are limited by resource availability, and $93 \%$ of otolaryngology residents reported concerns regarding PPE shortages in a recent publication. ${ }^{4}$ Some hospitals have requested the re-use of N95 masks, and sterilization techniques have been described. ${ }^{23}$

It is thus heartening to note that more than $80 \%$ of respondents had been provided with adequate PPE. On the other hand, up to $50 \%$ of survey respondents indicated that they acquired their own PPE. We did not explore the reasons for this, but postulate that reasons may include differential hospital PPE guidelines, availability, and personal comfort level. From the results of our survey, it is apparent that safety is the top concern for trainees. Similar to prior survey findings, communication and transparency is critical to allay trainee concerns and impart a sense of control. ${ }^{4}$

In response to the pandemic, many institutions have rapidly transitioned to use of technology for delivery of didactic or interactive lectures, including strategies such as "flipped classroom", social media-based platforms with online practice questions, and teleconferences. ${ }^{7,24}$ Examples of tremendous collaborative efforts to supplement otolaryngology resident education include the Collaborative Multi-Institutional Otolaryngology Residency Education Program (CMIOREP, University of Southern California), ${ }^{25}$ Great Lakes Otolaryngology Consortium (GLOC, Case Western Reserve University), ${ }^{26}$ and the Consortium of Resident Otolaryngology kNowledge Attainment (CORONA, University of Kentucky). ${ }^{27}$ These programs boast wide participation-CORONA, for example, includes 42 collaborator institutions, and reports over 400 daily participants globally.

Interestingly, we found that trainees in high COVID-19 prevalence regions had a greater increase in didactic activities, more often had a required curriculum and felt technology was used to good effect for educational activities. Accordingly, we found that trainees whose programs utilized technology well, had fewer concerns in regards to obtaining adequate education knowledge. These differences may reflect that regions with high COVID-19 prevalence were prescient towards the education needs during this changing environment, and implemented curricular changes at an earlier time. We also found there was a trend towards junior residents expressing greater concern towards their ability to receive adequate training in clinical and surgical experiences. This may reflect preferential case coverage by senior residents, or concern about the effects of the pandemic lasting longer than these few months, potentially impact their training in the long term. While increased didactic activities had an impact in allaying concerns about adequate educational knowledge, the concerns regarding adequate surgical training were prevalent across all levels and regions. In the setting of limited clinical operations, surgical training may require supplements through developing virtual simulationbased training, cadaver courses, and increased involvement of trainees in the operating room when restrictions 
ease.

Trainee responses were heterogeneous regarding research productivity. Various national research institutions including National Institute for Health Research (NIHR), biotechnology, and pharmaceutical companies across the world have had to suspend clinical trials, ${ }^{28,29}$ and research laboratories across the country have likewise suspended non-essential research. As expected, trainees in our study pursuing laboratory based and research requiring contact with clinical research subjects felt a decrease in their ability to continue research work, but others were able to increase clinical research activities. For surgical trainees who have seen their clinical volumes reduced, devoting time to clinical research may represent a valuable opportunity to develop an important skill set during this time.

Lastly, otolaryngology trainees are looking to begin their future careers at a time when companies conduct massive layoffs and many hospitals are imposing hiring freezes. ${ }^{30}$ Our study found a majority of senior trainees felt a negative impact on their ability to secure a job or fellowship at the completion of training. Some decided to "choose (a) job that I wouldn't have necessarily chosen" or defer entering the job market while entertaining the idea of further training to "increase certain exposure of surgical cases". Several respondents reflected that private physicians with a career performing elective surgeries may be more affected by a pandemic and economic pressures, and have begun rethinking their career goals. As a major goal of training is preparing for the next steps in future career, training programs will need to consider how to support their senior level trainees during this time of uncertainty.

\section{Conclusions}

The COVID-19 pandemic has challenged our community to consider how we balance our needs for educating trainees for future practice, and our call to duty to conserve resources in fighting this global pandemic as physicians. As take small steps forward in reopening clinical operations, additional limits, safety precautions and social distancing measures are likely to continue in the near future. We show that use of technology can mitigate some of the negative impact of decreased clinical exposure, and in the era of social distancing programs should continue develop virtual curriculums for training education. However, there is still room for improvement in ensuring trainees receive adequate surgical training to prepare for their future careers during this pandemic.

\section{References}

1. American College of Surgeons. COVID-19: guidance for Triage of Non-Emergent Surgical Procedures. March 17, 2020. Accessed April 21, 2020.

2. Patel RJ, Kejner A, McMullen C. Early institutional head and neck oncologic and microvascular surgery practice patterns across the United States during the SARS-CoV-2 (COVID19) pandemic. Head Neck. 2020.

3. Park JS, El-Sayed IH, Young VN, Pletcher SD. Development of clinical care guidelines for faculty and residents in the era of COVID-19. Head Neck. 2020.

4. Cai Y, Jiam NT, Wai KC, Shuman EA, Roland LT, Chang JL. Otolaryngology Resident Practices and Perceptions in the Initial Phase of the U.S. COVID-19 Pandemic. Laryngoscope. 2020.

5. Saibene AM, Allevi F, Biglioli F, Felisati G. Role and Management of a Head and Neck Department during the COVID-19 Outbreak in Lombardy. Otolaryngol Head Neck Surg. 2020:194599820917914.

6. Potts JR, 3rd. Residency and Fellowship Program Accreditation: Effects of the Novel Coronavirus (COVID-19) Pandemic. J Am Coll Surg. 2020.

7. Crosby DL, Sharma A. Insights on Otolaryngology Residency Training during the COVID-19 Pandemic. Otolaryngol Head Neck Surg.2020:194599820922502.

8. Shah JP. The impact of COVID-19 on Head and Neck surgery, education, and training. Head Neck. 2020.

9. Surgery AAoO-HN. Academy Supports CMS, Offers Specific Nasal Policy. . March 26, 2020. 
10. Vukkadala N, Qian ZJ, Holsinger FC, Patel ZM, Rosenthal E. COVID-19 and the Otolaryngologist: Preliminary Evidence-Based Review.Laryngoscope. 2020.

11. Tay JK, Khoo ML, Loh WS. Surgical Considerations for Tracheostomy During the COVID-19 Pandemic: Lessons Learned From the Severe Acute Respiratory Syndrome Outbreak. JAMA Otolaryngol Head Neck Surg.2020.

12. Eva KW, Anderson MB. Medical Education Adaptations: Really Good Stuff for educational transition during a pandemic. Med Educ.2020.

13. Shah JP. Global On line fellowship. JACS. 2020.

14. Adams JM. March 14, 2020; https://twitter.com/surgeon_gen$\mathrm{eral} / \mathrm{status} / 1238798972501852160$ ?lang $=\mathrm{en}$.

15. Givi B. Advanced Head and Neck Surgery Training during the COVID-19 Pandemic. Head Neck. May $8,2020$.

16. ACGME. ACGME Statement on the Shortage of Personal Protective Equipment (PPE). https://acgme.org/Newsroom/Newsroom-Details/ArticleID/10186/ACGME-Statement-on-the-Shortage-ofPersonal-Protective-Equipment-PPE. Accessed May 7, 2020.

17. Shetty K, Poo SXW, Sriskandarajah K, et al. "The Longest Way Round Is The Shortest Way Home": An Overhaul of Surgical Ward Rounds. World J Surg. 2018;42(4):937-949.

18. Montague ML, Lee MS, Hussain SS. Staff attitudes to a daily otolaryngology ward round. J Laryngol Otol. 2004;118(12):963-971.

19. Chan JYK, Tsang RKY, Yeung KW, et al. There is no routine head and neck exam during the COVID-19 pandemic. Head Neck. 2020.

20. Simonds AK, Sokol DK. Lives on the line? Ethics and practicalities of duty of care in pandemics and disasters. Eur Respir J.2009;34(2):303-309.

21. American College of Surgeons. COVID-19: Considerations for Optimum Surgeon Protection. . https://www.ahns.info/wp-content/uploads/2020/04/considerations_optimum_surgeon_protection.pdf.

22. ENTUK. ENTUK Guidelines for changes in ENT during COVID-19 Pandemic. https://www.entuk.org/entuk-guidelines-changes-ent-during-covid-19-pandemic. Accessed April 27, 2020.

23. de Man P, van Straten B, van den Dobbelsteen J, van der Eijk A, Horeman T, Koeleman H. Sterilization of disposable face masks by means of standardized dry and steam sterilization processes; an alternative in the fight against mask shortages due to COVID-19. J Hosp Infect.2020.

24. Chick RC, Clifton GT, Peace KM, et al. Using Technology to Maintain the Education of Residents During the COVID-19 Pandemic. J Surg Educ. 2020.

25. Collaborative Multi-Institutional Otolaryngology Residency Education Program (CMIOREP) https://sites.usc.edu/ohnscovid/. Accessed April 21, 2020.

26. Great Lakes Otolaryngology Consortium (GLOC). http://www.uhhospitals.org/ENTEDConsortium. Accessed April 28, 2020.

27. Consortium Of Resident Otolaryngologic kNowledge Attainment (CORONA) Initiative in Otolaryngology. https://entcovid.med.uky.edu/. Accessed April 25, 2020.

28. Feuerstein A. As Covid-19 spreads, disruptions to clinical trial and drug development accelerate. Stat News March 23, 2020. 
29. Research. NIfH. DHSC issues guidance on the impact of COVID-19 on research funded or supported by NIHR. . March 16, 2020; https://www.nihr.ac.uk/news/dhsc-issues-guidance-on-the-impact-on-covid-19on-research-funded-or-supported-by-nihr/24469. Accessed April 14, 2020.

30. Yaffe-Bellany DP, Jaclyn. For the Class of 2020, a Job-Eating Virus Recalls the Great Recession. New York Times March 27, 2020.

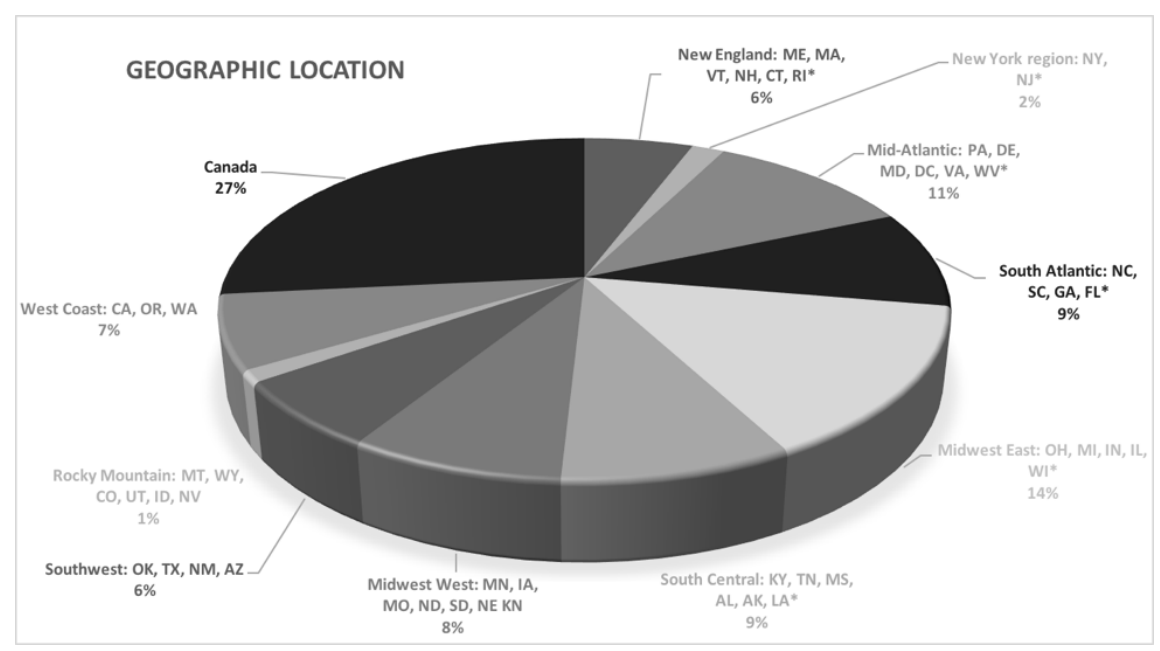

Figure 1: This is a caption

\section{Hosted file}

Tables_COVID_trainee_HED-20-1112.docx available at https://authorea.com/users/312328/ articles/461372-impact-of-the-covid-19-pandemic-on-otolaryngology-trainee-education

\section{Hosted file}

Supplemental_Table_1_HED-20-1112.docx available at https://authorea.com/users/312328/ articles/461372-impact-of-the-covid-19-pandemic-on-otolaryngology-trainee-education 